\title{
EDITORIAL
}

\section{Man versus microbe: warfare at its worst}

\author{
The UK Five Year Antimicrobial Resistance Strategy report calls for an integrated response \\ from the academic, pharmaceutical and political sectors to combat antibiotic resistance.
}

In March 2013, the second volume of the Annual Health Report of the Chief Medical Officer for England was published, a major component of which was a welcome focus on antimicrobial resistance (AMR). The report detailed the key challenges associated with AMR, and a follow-up report setting out a $\underline{5-y e a r}$ strategy plan has now been published. The strategy emphasizes the need for a collaborative and committed effort from academia, the medical and veterinary sectors, the pharmaceutical industry and both the UK and international governments. In addition, it contains a call for action in seven key areas to improve our understanding of the emergence and spread of AMR, to ensure more effective stewarding of existing antibiotics and to re-invigorate the diminishing antibiotic pipeline.

Better access to, and use of, surveillance data is emphasized as a crucial means to increase our knowledge of resistance trends, the epidemiology of infections, drug use and clinical outcomes, which should facilitate the development and implementation of policies for the rational prescription of antibiotics in both human and animal healthcare. The importance of improved diagnostic tools - which are needed to determine resistance profiles to inform the appropriate use of drugs - is also highlighted as key to achieving this goal. Such tools should also reduce the widespread overuse and misuse of our current antibiotic arsenal, together with more effective antibiotic stewardship, including greater adherence to antibiotic guidelines in the agricultural sector. However, stewardship is dependent on knowing what appropriate and responsible antibiotic use actually means and, in practical terms, how much of a drug is needed and for how long. These are complicated questions that lack clear answers, and future research is needed to clarify and standardize such issues.

It is more than 20 years since a new class of antibiotics entered the market. The establishment of a diverse and sustainable pipeline of new drugs is paramount, and a lack of incentivization of the pharmaceutical industry is one of the primary obstacles to achieving this goal. Antibiotic discovery is an arduous task that is plagued by difficulties in the identification and development of novel compounds, in addition to the unfavourable economics of antibacterial drug development. It can take up to 20 years from target discovery to introduce a new drug to market, at costs that often run into hundreds of millions of pounds. This is compounded by the fact that new drugs will have a shortened shelf-life if they are widely used and will therefore have a highly restrictive usage tag, which means little or no financial return on investment. Thus, changes in the drug development paradigm are urgently needed, and the report highlights the potential of public-private partnerships to effect such change. One promising venture is the Innovative Medicines Initiative consortium New Drugs for Bad Bugs (ND4BB), which brings academic and pharmaceutical partners together to work towards the development of new antibiotics for drug-resistant pathogens. It involves a multitiered framework that aims not only to foster the discovery and development of new drugs but also to design and implement new business models. This multistakeholder approach has proved to be successful in restoring pharmaceutical engagement, as exemplified by the Medicines for Malaria Venture (MMV), which is a non-profit public-private partnership that was established in 1999, when industry interest in the development of new antimalarial drugs had declined. One notable achievement of MMV has been the introduction of the drug Coartem Dispersible (MMV and Novartis; artemether and lumefantrine) for the treatment of paediatric malaria. Finally, although the strategy report highlights that funding for translational research is a priority, the importance of basic research is notably absent. Clearly, with advances in high-throughput technologies, particularly in the genomics field, combined with progress in synthetic biology, basic science will continue to be a major contributor to the discovery and development of antibiotics and should be a funding priority.

Just over a decade ago, the late Nobel laureate Joshua Lederberg wrote that "the future of humanity and microbes likely will unfold as episodes of a suspense thriller that could be titled 'Our Wits Versus Their Genes.” (REF. 1). Lederberg's analogy captures the ongoing battle between man and microbes and, by this point in the 'box set', mankind seems to have been outwitted. The publication of the strategy report has been met with a strong sense of optimism and a collective will to execute the necessary interventions, but this is not a storyline that has a speedy resolution. All engaged parties should be encouraged to maintain the current energy and enthusiasm to ensure that momentum is sustained. 1. Lederberg, J. Science 288, 287-293 (2000). 